\title{
Integral-stiffness-based Optimization Method for Designing a Computer Numerically Controlled Grinding Machine
}

\author{
Kun-Chieh Wang, ${ }^{*}$ Chi-Hsin Yang, Long Wu, Zijian Ai, and Hai-Lian Hong \\ School of Mechanical and Electric Engineering, Sanming University, Sanming, Fujian Province 365004, China
}

(Received October 21, 2020; accepted March 22, 2021)

Keywords: optimal structure design, machine tools, finite element analysis, grinding machine, vibration sensor, displacement sensor, machine stiffness

New methods of optimizing the design of machines with high stiffness have attracted much attention. Conventionally, machine designers have carried out optimization by attempting to minimize static deformation or maximize static stiffness. Nevertheless, the dynamic behavior of the machine structure plays a deterministic role in the final machining precision. Therefore, we propose in this study an integral-stiffness-based optimization method for designing the optimal structure of a computer numerically controlled (CNC) grinding machine. The proposed novel optimization methodology includes a prototype designed on the basis of know-how and the determination of control parameters based on the mode shape, Taguchi's experimental method based on finite element analysis (FEA), and grey relational analysis (GRA). The target parameters in the optimization are static stiffness, first natural frequency, and dynamic stiffness. Results reveal that the optimal structure of a CNC grinding machine obtained by merely considering the static stiffness exhibits good performance when applying static forces but inferior performance when applying dynamic forces. A good optimization approach for designing a high-precision machine should integrally consider the static stiffness as well as the dynamic stiffness. With our proposed methodology, machine designers can design an optimal high-stiffness structure of a CNC grinding machine more efficiently and accurately.

\section{Introduction}

Because of the increasingly strict requirements on the machining accuracy, reliability, and stability of tool machinery, the goal of optimization for machine structures has gradually shifted from high static stiffness towards high dynamic stiffness, and subsequently to high integral stiffness (both static and dynamic stiffness). Many previous studies on the optimization of machine structures focused on a single module or major components such as the spindle, bed, or column, ${ }^{(1-3)}$ or adopted conventional methods of structure analogy, experience-based design, or optimization considering only static stiffness, ${ }^{(4-7)}$ which have thus far been insufficient and inaccurate. Moreover, numerous optimization methods for increasing the stiffness of the overall structure of a machine tool or its components have been proposed. For example, Lei et al. ${ }^{(8)}$

*Corresponding author: e-mail: m18316252102@126.com https://doi.org/10.18494/SAM.2021.3181 
proposed an optimization method based on sensitivity analysis for designing lightweight machine tools. Chen et al. ${ }^{(9)}$ studied the optimization problem of a vertical machining center using a response surface method. Liusheng et al. ${ }^{(10)}$ adopted topology optimization to improve the stiffness of the rib plate in a machine's column. Jie ${ }^{(11)}$ investigated the optimization problem of a lathe bed using dynamic characteristic analysis. From the development history of the above proposed optimization techniques for the overall structure of a machine or its components, it can be seen that consideration of a machine's dynamic behavior is not only a trend but also a prerequisite in the accurate design of machine tools with an optimal structure.

For computer numerically controlled (CNC) machine tools, the static and dynamic stiffness are the most important and fundamental parameters that determine their machining performance. The static stiffness refers to the resistance to deformation when a force is applied on the machine structure. The natural frequencies and their corresponding modal shapes of free oscillation provide an insight into the resonance of the machine. The dynamic stiffness is directly related to the dynamic response (deformation) of the machine structure during the application of external periodic forces. These three parameters are strongly connected with each other. Sometimes a structure with good static stiffness still exhibits large deformation or even damage due to resonance or dynamic stimuli at certain frequencies. Thus, an integrative examination of the influence of these parameters should be made to improve the design of a machine's structure. In this regard, we will introduce the concept of integral stiffness in the optimization process, which refers to a combination of static and dynamic stiffness.

Furthermore, in the optimization process, too many experiments must be performed to consider the large number of possible variations of variables. To overcome this problem, we adopt a popular experimental method called Taguchi's method, ${ }^{(12)}$ which can effectively reduce the number of experiments and still capture the influence of controlled variables on target variables. Moreover, to verify the results of finite element analysis (FEA), experiments on measuring the static deformation of a machine structure via displacement sensors are also performed in this study.

In this study, we propose an innovative methodology based on integral stiffness to optimize the structure of a CNC machine tool. In the proposed methodology, we start from a prototype machine designed on the basis of know-how and experience. Then, integral optimization schemes, which include FEA, experimental identification, parameter selection based on mode shapes, Taguchi's method, grey relational analysis (GRA), and experimental verification, are applied to completely and accurately obtain the optimal structure of a machine tool.

\section{Integral-stiffness-based Optimization Scheme}

Our proposed integral optimization scheme mainly comprises eight steps, as shown in Fig. 1. First, we choose a CNC three-head grinding machine as the target for illustration since its structure is sophisticated and complex. This target machine features a long and narrow base, a wide column, and a three-head ram. Its structure is weak and deforms largely during machining, and thus it provides a severe test for the proposed novel methodology. Second, we design a prototype grinding machine based on accumulated know-how and experience. Third, we 


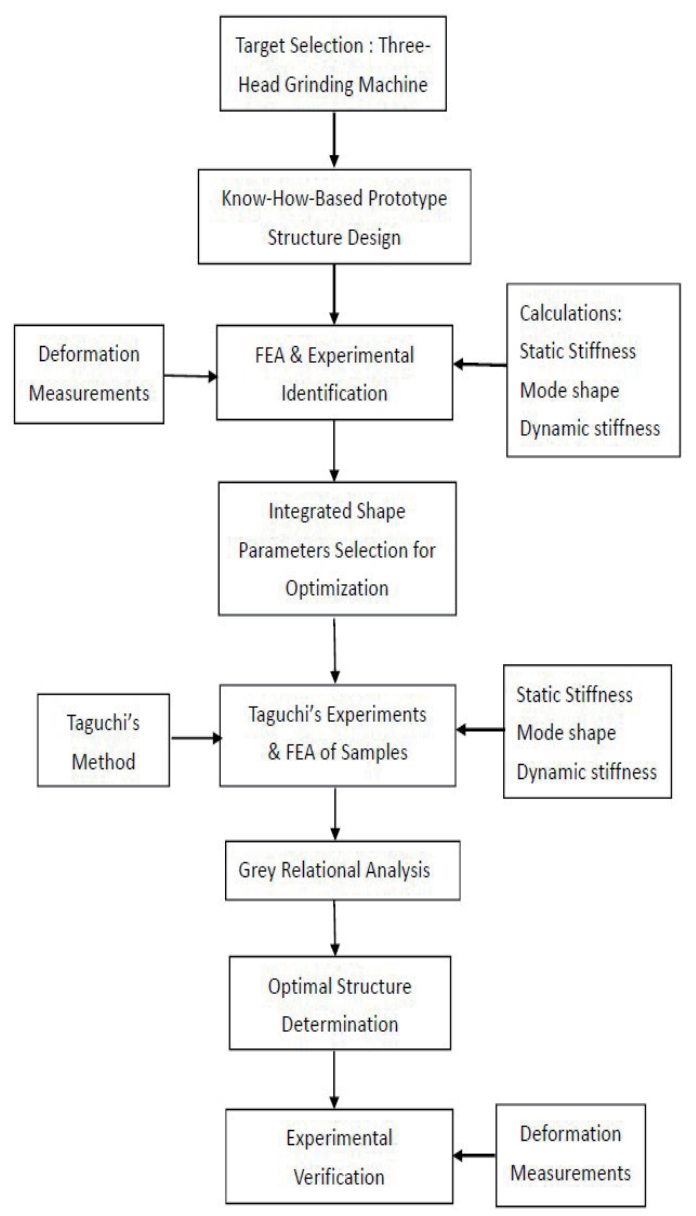

Fig. 1. Flow chart of proposed integral optimization scheme.

examine three aspects of the behavior of the target machine structure using FEA: static stiffness, vibration mode shape, and dynamic stiffness. Moreover, to identify the machine's static deformation, a measurement using displacement sensors is conducted. Fourth, we analyze the calculated and measured results obtained in the third step to acquire shape parameters that enhance the structure's integral stiffness. Fifth, we construct a special set of orthogonal arrays (OAs) for Taguchi's experiment based on FEA to investigate the influence of changing these shape parameters on integral stiffness. Sixth, we analyze these results via GRA to obtain an optimal set of shape parameters. Seventh, we construct an optimal machine structure and perform a related FEA calculation. Eighth, we verify the optimal structure via experimental measurements of the machine's static deformation.

\section{Theoretical Foundations}

In our proposed integral optimization scheme, the related theories comprise (1) solid mechanics: static mechanics, vibration mechanics, and dynamic response analysis; (2) experiment design: Taguchi's method; (3) finite element method (FEM); (4) optimization strategy. The following section gives a brief introduction to these theories. 


\subsection{FEA}

\subsubsection{FEM}

The FEM is a popular and efficient way to analyze a machine's structure. ${ }^{(13)}$ All linear or nonlinear stress problems in solid mechanics, heat transfer, and fluid dynamics can be analyzed using FEM. FEM has been successfully applied in many areas, ${ }^{(14-17)}$ and many FEM programs have been developed, e.g., ANSYS, SOLIDWORKS, NASTRAN, CREO, and INVENTOR. ${ }^{(18,19)}$ SOLIDWORKS has the feature that one may directly use the constructed 3D sketch of the target product to perform FEA. Therefore, we adopt this software program to perform the FEM calculation in this study.

Many previous studies have examined machine stiffness using numerical methods. ${ }^{(20-22)}$ Vivekananda et al. ${ }^{(23)}$ adopted ANSYS software to obtain natural frequencies of a machine tool. Hong et al. ${ }^{(24)}$ investigated the static structure of a five-axis machining center using SOLIDWORKS. Wang(25) used SOLIDWORKS to investigate the structure stiffness of a compound machining center.

The procedure of FEM using SOLIDWORKS includes the following three steps. (1) Preprocessing phase: dividing the solution domain into suitable finite elements, determining shape functions, developing governing equations for individual elements, assembling and constructing the global stiffness matrix, and applying suitable restricted conditions. (2) Solution phase: solving equations to obtain nodal results (displacement, stress, and strain). (3) Postprocessing phase: calculating other variables (natural frequencies and stiffness).

\subsubsection{Static mechanics}

When a load is applied on a given body, the static equation of displacement can be expressed as

$$
[K]\{X\}=\{F\},
$$

or

$$
[K]\{X\}=\left\{F_{e}\right\}+\left\{F_{r}\right\},
$$

where $[K]=\sum_{i=1}^{N}\left[K_{S}\right]$ is the stiffness matrix of the system, $\{X\}$ is the displacement vector, $N$ is the element number, $\left[K_{s}\right]$ is the element stiffness matrix, $\left[F_{e}\right]$ is the total external force vector, and $\left[F_{r}\right]$ is the reaction load vector.

\subsubsection{Modal analysis}

Modal analysis is mainly concerned with the calculation of natural frequencies and mode shapes of a structure. Note that three assumptions are usually made in modal analysis: no 
damping effect, a linear structure, and none of the physical properties change with time (e.g., force and displacement). Since no actual force is applied to the structure, the structure is under free vibration. The equation of free vibration is

$$
[M]\{\ddot{X}\}+[K]\{X\}=\{0\} .
$$

Since the structure is linear, the motion is harmonic. Thus, we may assume that the displacement has the following form:

$$
\{X\}=\{X\}_{i} e^{j \omega_{i} t}, i=1,2, \ldots, n,
$$

where $[X]_{i}$ denotes the amplitude (mode shape) for the $i$ th frequency $\omega_{i}$ and $n$ is the number of degrees of freedom of the structure. Then, we substitute Eq. (4) into Eq. (3) and obtain

$$
\left[[K]-\omega_{i}^{2}[M]\right]\{X\}_{i}=\{0\} .
$$

Equation (5) is an eigenvalue problem. Non-trivial solutions occur only under the following criterion:

$$
\operatorname{det}\left(\left[[K]-\omega_{i}^{2}[M]\right]\right)=0 .
$$

We solve the above equation to obtain the eigenvalues $\left\{\omega_{i}\right\}$ and their corresponding eigenvectors $[X]_{i}$.

\subsubsection{Dynamic response}

When a time-variant force is applied to a structure, the governing equation of its dynamic response can be represented as

$$
[M]\{\ddot{X}\}+[C]\{\dot{X}\}+[K]\{X\}=\{F(t)\}
$$

where $[M]$ and $[K]$ were defined previously, $[C]$ is the damping matrix, and $\{F(t)\}$ is the timedependent external load vector. Here, we set

$$
\{F(t)\}=\left\{F_{\max } e^{i \Psi}\right\} e^{i \omega t}
$$

and

$$
\{X\}=\left\{X_{\max } e^{i \phi \varphi} e^{i \omega t}\right\},
$$

where $F_{\max }$ denotes the maximum absolute value of the external force, $\Psi$ denotes the force phase angle, $X_{\max }$ denotes the maximum absolute value of the displacement, and $\phi$ denotes the 
displacement phase angle. Substituting Eqs. (8) and (9) into Eq. (7) and rearranging, we obtain the following final equation for the structural dynamic response:

$$
\left(-\omega^{2}[M]+i \omega[C]+[K]\right)\left\{X_{\max } e^{i \phi}\right\}\left\{F_{\max } e^{i \Psi}\right\}
$$

The desired displacement vector of the dynamic response $\left\{X_{\max } e^{i \phi}\right\}$ can be obtained from Eq. (10).

\subsection{Taguchi's method}

Experimental studies usually involve a significant number of factors, and full factorial design results may involve a large number of experiments. To reduce the number of experiments to a practically acceptable level, we need a good selection method. Here, we adopt Taguchi's method, ${ }^{(26)}$ which is a popular method for reducing the number of experiments in factorial design. The method of selecting a limited number of experiments that provide the most information is known as a partial factorial experiment (PFE). Taguchi constructed a special set of OAs to select PFEs. A single OA may accommodate a number of experimental conditions. Commonly used OAs have two, three, or even four levels. Taguchi's method includes four steps. 1. Determine the quality characteristics and design parameters related to the product or process. 2. Design and conduct the experiments. 3. Analyze the results to determine the optimal conditions. 4. Run a confirmatory test using the optimal conditions.

\subsection{GRA}

$\mathrm{GRA}^{(27)}$ is a method of measuring the correlation between discrete data sequences. Compared with the conventional regression method, GRA has the advantages of no restriction on the amount or distribution of data. GRA is a good choice for dealing with problems where the amount of data is insufficient or the data do not follow normal distributions. The steps of GRA are as follows.

Step 1. Construct an initial data sequence from the collected data,

$$
\left\{\begin{array}{c}
x_{1}^{(0)}=\left\{x_{1}^{(0)}(1), x_{2}^{(0)}(2), \ldots, x_{1}^{(0)}(m)\right\} \\
x_{2}^{(0)}=\left\{x_{2}^{(0)}(1), x_{2}^{(0)}(2), \ldots, x_{2}^{(0)}(m)\right\} \\
\vdots \\
x_{n}^{(0)}=\left\{x_{n}^{(0)}(1), x_{n}^{(0)}(2), \ldots, x_{n}^{(0)}(m)\right\}
\end{array} .\right.
$$

Step 2. Normalize the initial data sequence using the following equation:

$$
x_{i}^{*}(k)=\frac{x_{i}^{(0)}(k)}{\xi}, i=1,2, \ldots, n ; k=1,2, \ldots, m,
$$

where $\xi=x_{i}^{(0)}(1)$ is chosen as the initial data and $X_{i}^{*}=\left\{x_{i}^{*}(k)\right\}$. 
Step 3. Choose an ideal data sequence $X_{0}^{*}$ (usually the worse or the best) from $X_{i}^{*}$ as the reference data sequence, denoted as

$$
X_{0}^{*}(k)=\left\{x_{0}^{*}(1), x_{0}^{*}(2), \ldots, x_{0}^{*}(m)\right\}
$$

Step 4. Calculate the norm sequence $\Gamma_{i}=\left\{\Delta_{0 i}(1), \Delta_{0 i}(2), \ldots, \Delta_{0 i}(m)\right\}$, whose elements are the absolute values of the differences between the comparison sequence and reference sequence, defined as

$$
\Delta_{0 i}(k)=x_{0}^{*}(k)-x_{i}^{*}(k) \|
$$

Step 5. Find the maximum and minimum norms among all difference sequences using the following equations:

$$
\alpha=\max _{i=1}^{n} \max _{k=1}^{m} \Delta_{0 i}(k), \beta=\min _{i=1}^{n} \min _{k=1}^{m} \Delta_{0 i}(k) .
$$

Step 6. Calculate the grey relational coefficients as follows:

$$
\gamma_{0 i}(k)=\frac{\beta+\lambda \cdot \alpha}{\Delta_{0 i}(k)+\lambda \cdot \alpha}, \lambda \in[0,1]
$$

In the above equation, $\lambda$ is called the distinguishing coefficient. It represents the contrast between the background and measured data. In general, it is set as $\lambda=0.5$.

\section{Results and Discussion}

\subsection{Prototype design}

The prototype of our target three-head grinding machine was designed by professional designers of $\mathrm{CNC}$ machine tools with over 20 years of experience in designing machine tools, particularly grinding machines. The thus designed prototype structure based on accumulated know-how and market needs is called Case 1 and shown in Fig. 2.

\subsection{Static and dynamic structural behavior of prototype}

Using FEA, we examine three aspects of the structure behavior of the prototype machine: static stiffness, vibration mode shape, and dynamic stiffness. In addition, to determine the difference between actual and simulation results, the static deformation and mode shape of the machine are measured for comparison. 


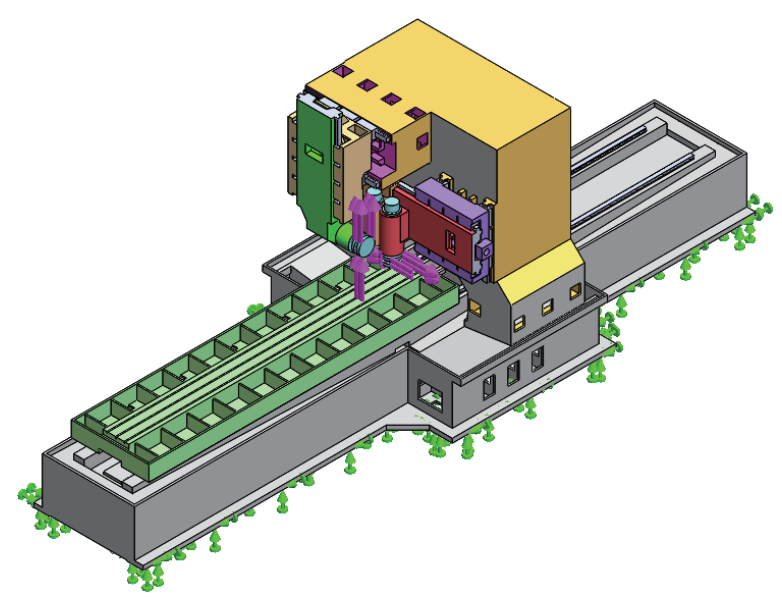

Fig. 2. (Color online) Configuration and restricted conditions of prototype machine.

\subsubsection{Restricted conditions and grid-independent test}

The applied forces are set to act at the nose of the left, right, and upper grinding heads in the $Y,-Y$, and $Z$ directions, respectively, with a magnitude of $100 \mathrm{~kg}_{\mathrm{f}}$. The structure material is designated as graphite cast iron. Then, four different meshes are established with minimum element sizes of $10,6,4$, and $3 \mathrm{~mm}$, and it is found that the relative errors of the calculated first natural frequencies are $0.786,0.383,0.0324$, and $0.0331 \%$, respectively. Therefore, the element size of $4 \mathrm{~mm}$ is chosen for all the FEA calculations. For example, the mesh of the prototype has 125158 node numbers and 596491 elements.

\subsubsection{Static behavior-stress, strain, and displacement}

The static stress, strain, and displacement are calculated. The obtained displacement distribution of the prototype machine is shown in Fig. 3, which has a maximal displacement of $14 \mu \mathrm{m}$ occurring at the grinding head nose in the $Z$ direction (corresponding to a minimal static stiffness of $K_{s}=12.37 \mathrm{~kg}_{\mathrm{f}} / \mu \mathrm{m}$ ). Furthermore, the magnitude of the displacement of the module components decreases in the order head $>$ head ram $>$ cross column $>$ vertical column $>$ base. Because the module components of the head, head ram, and cross column have larger deformations than the other parts of the machine structure, we may choose the static stiffness of the grinding head nose, denoted by $K_{S}$ as one of the index parameters for optimization.

\subsubsection{Verification via displacement measurements using gauge sensors}

To identify the difference between the FEA results and the actual behaviors of the prototype machine, a displacement measurement experiment is carried out. Six strain gauge sensors are stamped on the front end of each grinding head to measure the displacement. We apply forces of $100 \mathrm{~kg}_{\mathrm{f}}$ in the $Y,-Y$, and $Z$ directions via the load cells at the front ends of the left, right, and 


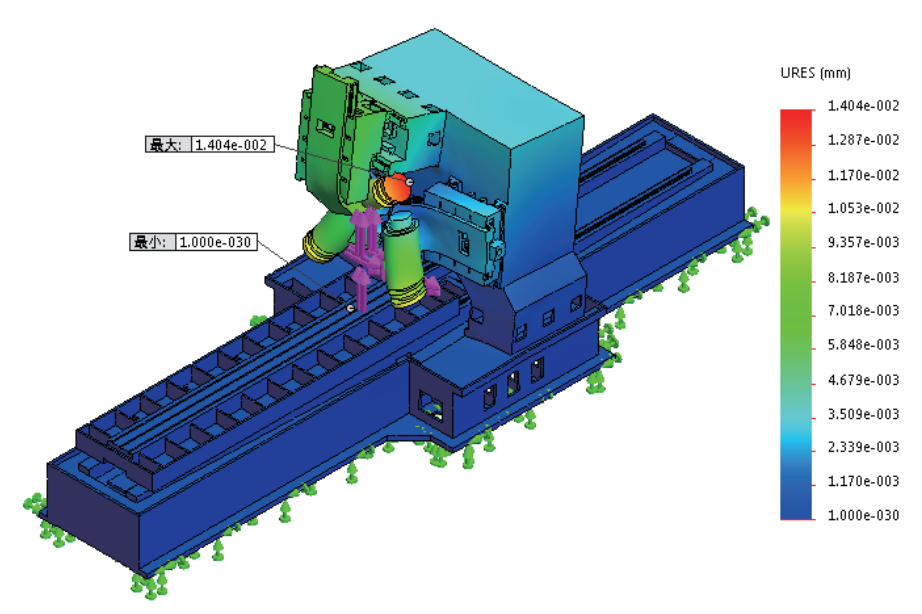

Fig. 3. (Color online) Displacement distribution of structure in Case 1.

upper grinding heads. Among the six displacement sensors, two of them are stamped on the upper grinding spindle, two on the right grinding spindle, and the other two on the left grinding spindle. The measured results show that the maximum deformation of $0.01426 \mathrm{~mm}$ occurs at the bottom of the upper grinding head, which corresponds to an error of $8.85 \%$ relative to the FEM simulation results. This shows that the following FEA simulation results only slightly deviate from the actual machine's behaviors.

\subsubsection{Mode shape analysis}

The dynamic responses consist of two phases: the natural vibration and the frequency response of the forced vibration. The mode shape analysis of natural vibration provides a clear insight into the effect of the bias of dynamic interaction on the interface between assemblies. Analyzing the types of mode shape of the natural vibration and investigating the fragile parts of the structure may suggest modifications to the dimensions to enhance the stiffness of the whole machine.

Using Eqs. (3)-(6), we obtain the natural frequencies of the target grinding machine. The first 30 natural frequencies (M1-M30) are listed in Table 1. Also, the first eight mode shapes are shown in Fig. 4, which are used for conveniently analyzing the dynamic behaviors of the machine. It can be seen from the first six mode shapes that the column deforms back and forth at $f=35.9 \mathrm{~Hz}$ and $f=55.5 \mathrm{~Hz}$, the ram deforms to the left and right at $f=83 \mathrm{~Hz}$, the column twists at $f=88.6 \mathrm{~Hz}$, and the head deforms at $f=107.6 \mathrm{~Hz}$ and $f=143.0 \mathrm{~Hz}$. By examining the deformation of these six mode shapes frequently encountered in machining, it is clarified that the dimensions of the column, head ram, and head, and the types of their inner ribs are crucial factors determining the occurrence of dynamic error of a machine. We set the structure parameters used in Taguchi's experiment as follows: $A$ : ram length (350 and $300 \mathrm{~mm}), B$ : ram width (250 and $200 \mathrm{~mm}), C$ : vertical column length $(855$ and $955 \mathrm{~mm}), D$ : vertical column width (500 and $600 \mathrm{~mm}), E$ : inner structure (rib) type ( + and $*), F$ : cross column height (925 and 
Table 1

First 30 natural frequencies of the prototype machine ( $f$ : frequency).

\begin{tabular}{cccccccc}
\hline Mode no. & $f(\mathrm{~Hz})$ & & Mode no. & $f(\mathrm{~Hz})$ & & Mode no. & $f(\mathrm{~Hz})$ \\
\cline { 1 - 2 } \cline { 7 - 8 } M1 & 35.9 & & M11 & 188.9 & & M21 & 282.0 \\
M2 & 55.5 & & M12 & 210.0 & & M22 & 286.0 \\
M3 & 83.0 & & M13 & 217.3 & & M23 & 290.3 \\
M4 & 88.6 & & M14 & 223.7 & & M24 & 293.9 \\
M5 & 107.6 & & M15 & 228.1 & & M25 & 297.7 \\
M6 & 143.0 & & M16 & 238.6 & & M26 & 311.3 \\
M7 & 146.0 & & M17 & 254.3 & & M27 & 317.6 \\
M8 & 149.1 & & M18 & 261.4 & & M28 & 323.3 \\
M9 & 156.3 & & M19 & 275.6 & & M29 & 331.1 \\
M10 & 170.2 & & M20 & 277.8 & & M30 & 334.6 \\
\hline
\end{tabular}

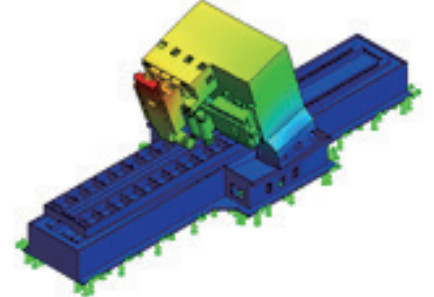

(a)

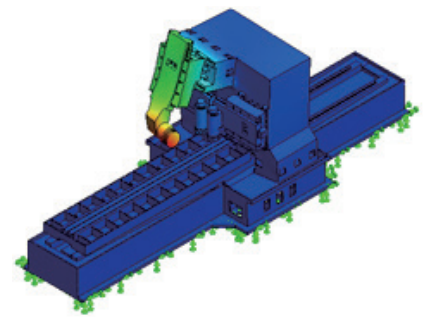

(c)

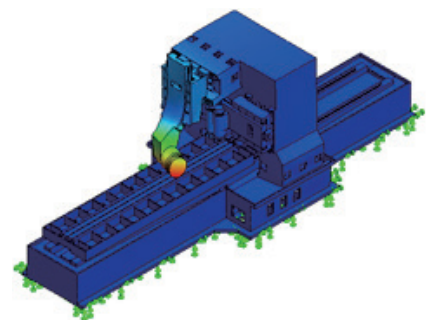

(e)

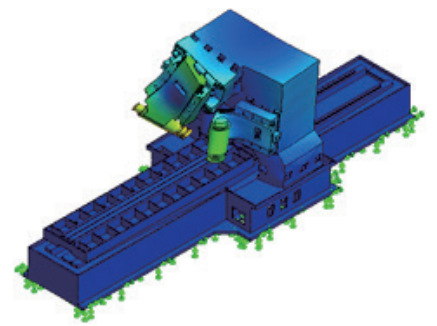

(g)

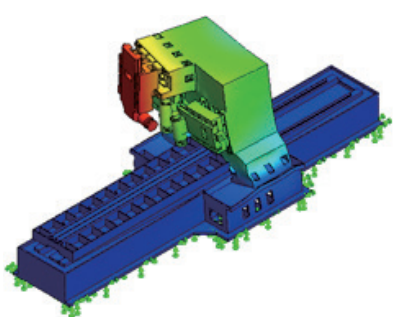

(b)

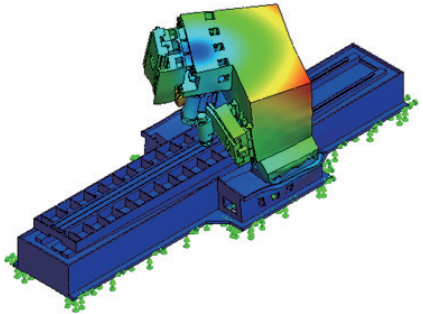

(d)

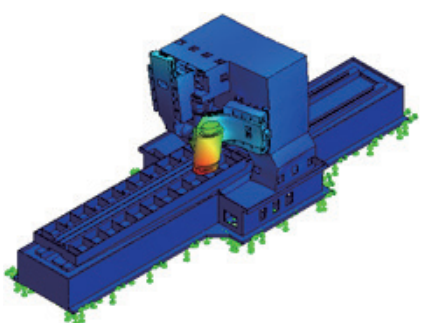

(f)

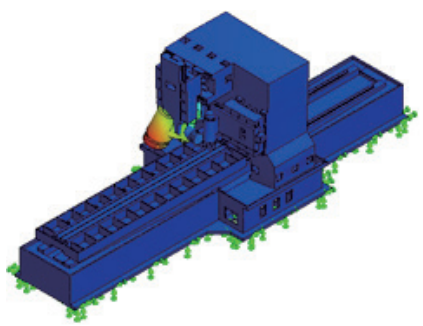

(h)

Fig. 4. (Color online) First eight mode shapes in Case 1. (a) M1 $f=35.9 \mathrm{~Hz}$. (b) M2 $f=55.5 \mathrm{~Hz}$. (c) M3 $f=83.0 \mathrm{~Hz}$. (d) M4 $f=88.6$ Hz. (e) M5 $f=107.6$ Hz. (f) M6 $f=143.0 \mathrm{~Hz}$. (g) M7 $f=146.0 \mathrm{~Hz}$. (h) M8 $f=149.1 \mathrm{~Hz}$. 
$1025 \mathrm{~mm}$ ), and $G$ : cross column length (1776 and $1926 \mathrm{~mm})$. Moreover, since the larger the first natural frequency the better, we choose it as one of the index parameters for optimization and denote it by $f_{s}$.

\subsubsection{Dynamic behavior}

A harmonic wave analysis is performed using Eqs. (7)-(10) to determine the dynamic response under the stimuli of periodically applied forces. The applied forces have the same magnitude as those in the static case $\left(100 \mathrm{~kg}_{\mathrm{f}}\right)$ but with harmonic frequencies ranging from 0 to $500 \mathrm{~Hz}$. The calculated maximal deformations for different frequencies are shown in Table 2. It can be seen that two large maximal displacements, $\delta_{1}=0.486 \mathrm{~mm}$ and $\delta_{2}=0.378 \mathrm{~mm}$, respectively appear at $f_{1}=35.9 \mathrm{~Hz}$ and $f_{2}=148.4 \mathrm{~Hz}$. The external stimuli at these two frequencies would induce a large deformation or failure during machining. The dynamic stiffness is then obtained at the nose of the upper grinding head via the formula $K_{d}=F / \delta_{1}$ and the obtained result is shown in Fig. 5. The minimal dynamic stiffness is $K_{d}=6.17 \times 10^{2} \mathrm{~kg}_{\mathrm{f}} / \mu \mathrm{m}$ at

Table 2

Maximal displacements for different frequencies in dynamic response of structure in Case 1.

\begin{tabular}{lccccccc}
\hline$f(\mathrm{~Hz})$ & $\delta(\mathrm{mm})$ & $f$ & $\delta$ & $f$ & $\delta$ & $f$ & $\delta$ \\
\hline $1.59 \mathrm{E}-06$ & 0.004 & 227.1 & 0.020 & 333.8 & 0.005 & 420.9 & 0.021 \\
35.9 & $\mathbf{0 . 4 8 6}$ & 236.1 & 0.017 & 339.7 & 0.001 & 425.5 & 0.025 \\
50.9 & 0.007 & 250.6 & 0.038 & 344.1 & 0.004 & 430.2 & 0.021 \\
76.5 & 0.009 & 259.7 & 0.007 & 352.0 & 0.003 & 436.9 & 0.031 \\
87.3 & 0.006 & 272.2 & 0.025 & 356.9 & 0.003 & 440.4 & 0.032 \\
103.2 & 0.016 & 277.3 & 0.036 & 360.4 & 0.003 & 450.5 & 0.006 \\
134.7 & 0.021 & 281.1 & 0.020 & 366.7 & 0.012 & 455.77 & 0.004 \\
145.3 & 0.084 & 285.0 & 0.019 & 369.0 & 0.020 & 462.1 & 0.003 \\
148.4 & 0.378 & 289.2 & 0.013 & 376.8 & 0.008 & 465.7 & 0.003 \\
154.6 & 0.069 & 293.0 & 0.016 & 379.6 & 0.016 & 469.3 & 0.003 \\
166.9 & 0.018 & 296.8 & 0.013 & 396.8 & 0.003 & 475.3 & 0.006 \\
184.5 & 0.008 & 308.2 & 0.005 & 403.2 & 0.004 & 480.2 & 0.004 \\
205.0 & 0.012 & 316.29 & 0.003 & 405.3 & 0.005 & 486.2 & 0.010 \\
215.6 & 0.048 & 321.9 & 0.003 & 407.0 & 0.005 & 491.7 & 0.040 \\
222.2 & 0.030 & 329.4 & 0.008 & 413.4 & 0.006 & 500.5 & 0.011 \\
\hline
\end{tabular}

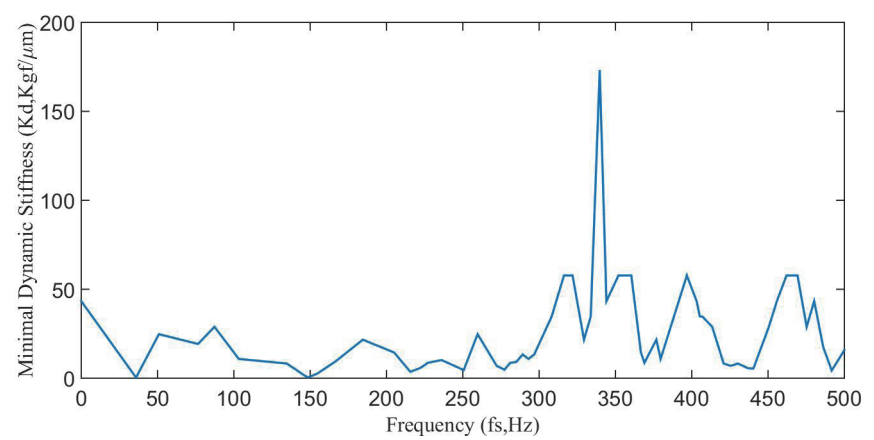

Fig. 5. (Color online) Minimal dynamic stiffness distribution of structure in Case 1. 
$35.9 \mathrm{~Hz}$. The strong and weak dynamic responses of the machine structure under external periodic stimuli can be clearly understood from this figure. Consequently, we may reasonably choose the minimal dynamic stiffness at the grinding head nose, denoted by $K_{d}$, as one of the index parameters for optimization.

\subsection{Determination of optimization parameters and Taguchi's experiment}

\subsubsection{Shape parameter selection}

By examining the static and dynamic conditions for which the target prototype machine is weak, we choose three index parameters as the decision factors for optimization: the minimal static stiffness at the nose $K_{s}$ (the larger the better), the first natural frequency $f_{s}$ (the larger the better), and the minimal dynamic stiffness $K_{d}$ (the larger the better). On the other hand, on account of possible dimension changes that induce large deformations occurring in the first six modal shapes, we select seven shape parameters, $A, B, C, D, E, F$, and $G$ (described previously), as the controlled factors. Each controlled factor has two variation levels. To reduce the number of experiments, we establish Taguchi's OA of L8 $\left(2^{7}\right)$ so that only eight experiments are required, as shown in Table 3.

\subsubsection{Optimization based on integral stiffness}

FEA calculations are performed for the above eight cases with $K_{s}, f_{s}$, and $K_{d}$ adopted as the index parameters. The calculation results are shown in Table 4.

\subsection{GRA for optimization}

To find the rankings of optimality for the above eight cases, we first normalize the obtained data in Table 4 via Eq. (12) and the result is shown in Table 5. Then we calculate their grey differences via Eq. (14) and the result is shown in Table 6. Finally, we obtain the grey relational coefficients using Eq. (16), which are given in Table 7. The results reveal that if we consider only the static stiffness of the machine structure, Case 5 has the highest ranking with static stiffness

\begin{tabular}{|c|c|c|c|c|c|c|c|}
\hline Cases & $A$ & $B$ & $C$ & $D$ & $E$ & $F$ & $G$ \\
\hline 1 & 300 & 200 & 855 & 500 & + & 925 & 1776 \\
\hline 2 & 300 & 200 & 855 & 600 & $*$ & 1025 & 1926 \\
\hline 3 & 300 & 250 & 955 & 500 & + & 1025 & 1926 \\
\hline 4 & 300 & 250 & 955 & 600 & $*$ & 925 & 1776 \\
\hline 5 & 350 & 200 & 955 & 500 & * & 925 & 1926 \\
\hline 6 & 350 & 200 & 955 & 600 & + & 1025 & 1776 \\
\hline 7 & 350 & 250 & 855 & 500 & $*$ & 1025 & 1776 \\
\hline 8 & 350 & 250 & 855 & 600 & + & 925 & 1926 \\
\hline
\end{tabular}


Table 4

FEA calculation results of $K_{s}, f_{s}$, and $K_{d}$ for cases 1 to 8.

\begin{tabular}{lccc}
\hline Cases & $K_{s}\left(\mathrm{~kg}_{\mathrm{f}} / \mu \mathrm{m}\right)$ & $f_{s}(\mathrm{~Hz})$ & $K_{d}\left(\mathrm{~kg}_{\mathrm{f}} / \mu \mathrm{m}\right)$ \\
\hline 1 & 12.340 & 35.900 & 0.356 \\
\hline 2 & 12.868 & 38.713 & 1.003 \\
\hline 3 & 12.826 & 37.983 & 0.941 \\
\hline 4 & 13.132 & 40.042 & 1.168 \\
\hline 5 & 13.152 & 41.235 & 1.035 \\
\hline 7 & 12.830 & 37.631 & 0.901 \\
\hline 8 & 12.596 & 37.113 & 0.512 \\
\hline
\end{tabular}

Table 5

Normalization results of optimization parameters for cases 1 to 8 .

\begin{tabular}{llll}
\hline Cases & $K_{S}\left(\mathrm{~kg}_{\mathrm{f}} / \mu \mathrm{m}\right)$ & $f_{s}(\mathrm{~Hz})$ & $K_{d}\left(\mathrm{~kg}_{\mathrm{f}} / \mu \mathrm{m}\right)$ \\
\hline 1 & 0.938 & 0.871 & 0.305 \\
\hline 2 & 0.978 & 0.939 & 0.859 \\
\hline 3 & 0.975 & 0.921 & 0.806 \\
\hline 5 & 0.998 & 0.971 & 1 \\
\hline 6 & 1 & 1 & 0.886 \\
\hline 7 & 0.976 & 0.913 & 0.771 \\
\hline 8 & 0.958 & 0.900 & 0.438 \\
\hline
\end{tabular}

Table 6

Grey differences for cases $1-8$.

\begin{tabular}{llll}
\hline Cases & $K_{S}\left(\mathrm{~kg}_{\mathrm{f}} / \mu \mathrm{m}\right)$ & $f_{s}(\mathrm{~Hz})$ & $K_{d}\left(\mathrm{~kg}_{\mathrm{f}} / \mu \mathrm{m}\right)$ \\
\hline 1 & 0.062 & 0.129 & 0.695 \\
\hline 2 & 0.022 & 0.061 & 0.141 \\
\hline 3 & 0.025 & 0.079 & 0.194 \\
\hline 5 & 0.002 & 0.029 & 0 \\
\hline 6 & 0 & 0 & 0.114 \\
\hline 7 & 0.024 & 0.087 & 0.229 \\
\hline 8 & 0.042 & 0.1 & 0.562 \\
\hline Grey difference & 0.072 & 0.184 & 0.703 \\
\hline
\end{tabular}

Table 7

Grey relational grades for cases $1-8$.

\begin{tabular}{lccccc}
\hline Cases & $K_{S}\left(\mathrm{~kg}_{\mathrm{f}} / \mu \mathrm{m}\right)$ & $f_{S}(\mathrm{~Hz})$ & $K_{d}\left(\mathrm{~kg}_{\mathrm{f}} / \mu \mathrm{m}\right)$ & $\begin{array}{c}\text { Grey relational } \\
\text { coefficient }\end{array}$ & $\begin{array}{c}\text { Grey relational } \\
\text { grade }\end{array}$ \\
\hline 1 & 0.89 & 0.795 & 0.418 & 0.701 & 7 \\
2 & 0.958 & 0.891 & 0.78 & 0.876 & 3 \\
3 & 0.952 & 0.864 & 0.72 & 0.845 & 4 \\
4 & 0.996 & 0.945 & 1 & 0.980 & 1 \\
5 & 1 & 1 & 0.814 & 0.938 & 2 \\
6 & 0.954 & 0.852 & 0.686 & 0.831 & 5 \\
7 & 0.922 & 0.833 & 0.471 & 0.742 & 6 \\
8 & 0.874 & 0.731 & 0.416 & 0.673 & 8 \\
\hline
\end{tabular}

$K_{s}=13.152 \mathrm{~kg}_{\mathrm{f}} / \mu \mathrm{m}$. Furthermore, if we consider the static stiffness (the larger the better) in conjunction with the first natural frequency (the larger the better) of the machine structure, Case 5 still has the highest ranking with $K_{s}=13.152 \mathrm{~kg}_{\mathrm{f}} / \mu \mathrm{m}$ and $f_{s}=41.235 \mathrm{~Hz}$. However, if we consider the static and dynamic stiffness simultaneously, Case 4 instead of Case 5 has the highest ranking with $K_{s}=13.132 \mathrm{~kg}_{\mathrm{f}} / \mu \mathrm{m}, f_{s}=40.042 \mathrm{~Hz}$, and $K_{d}=1.168 \mathrm{~kg}_{\mathrm{f}} / \mu \mathrm{m}$. This phenomenon that a machine structure with good static stiffness does not necessarily have good dynamic stiffness is highly noteworthy. Note that the dynamic stiffness of the machine structure is the factor that most strongly determines the machining precision. Therefore, to design a good machine structure, the simultaneous consideration of its static and dynamic stiffness is important. 


\subsection{Optimal structure, verification, and comparison}

The obtained first 30 natural frequencies and the corresponding first eight mode shapes of the optimal machine structure in Case 4 are shown in Table 8 and Fig. 6, respectively. It can be

Table 8

First 30 natural frequencies of the optimal machine in Case 4.

\begin{tabular}{cccccccc}
\hline Mode no. & $f(\mathrm{~Hz})$ & & Mode no. & $f(\mathrm{~Hz})$ & & Mode no. & $f(\mathrm{~Hz})$ \\
\cline { 1 - 4 } M1 & 40.0 & & M11 & 206.6 & & M21 & 282.5 \\
M2 & 60.5 & & M12 & 214.0 & & M22 & 289.2 \\
M3 & 87.0 & & M13 & 222.1 & & M23 & 291.3 \\
M4 & 91.0 & & M14 & 231.4 & & M24 & 294.5 \\
M5 & 108.1 & & M15 & 236.6 & & M25 & 298.7 \\
M6 & 145.8 & & M16 & 249.4 & & M26 & 305.2 \\
M7 & 146.8 & & M17 & 252.3 & & M27 & 309.3 \\
M8 & 150.6 & & M18 & 262.7 & & M28 & 311.5 \\
M9 & 154.3 & & M19 & 266.2 & & M29 & 320.6 \\
M10 & 170.5 & & M20 & 272.2 & & M30 & 336.1 \\
\hline
\end{tabular}

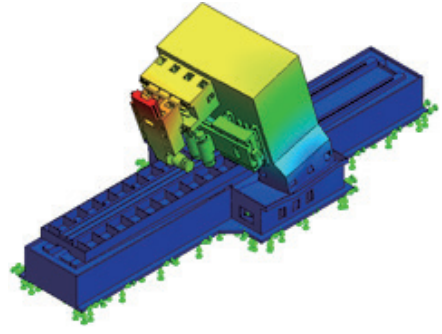

(a)

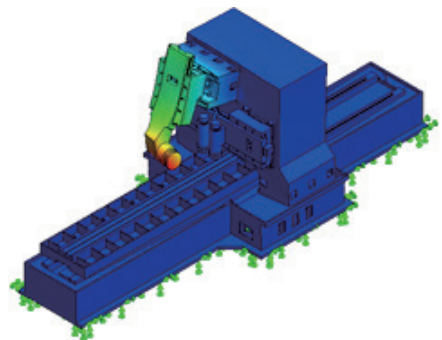

(c)

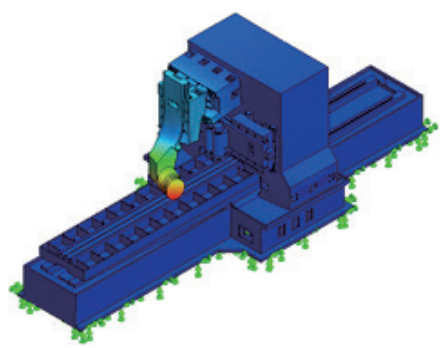

(e)

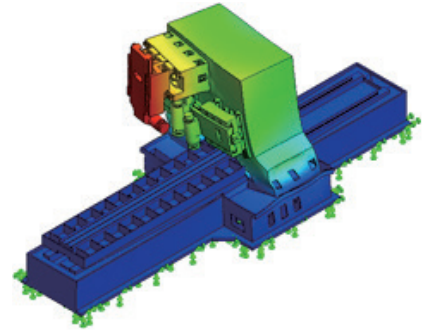

(b)

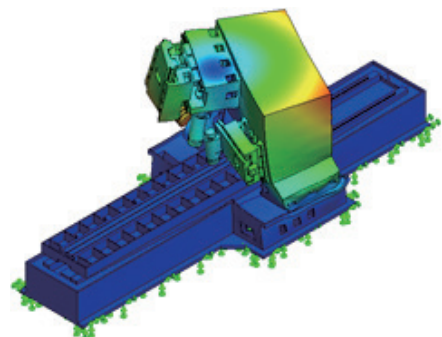

(d)

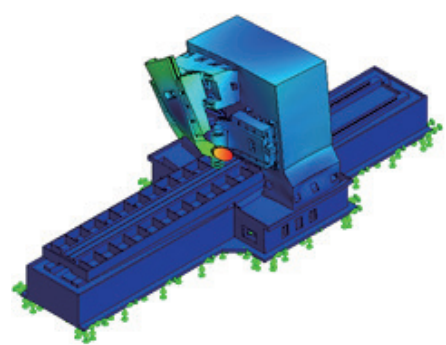

(f)

Fig. 6. (Color online) First eight mode shapes in Case 4. (a) M1 $f=40.0 \mathrm{~Hz}$. (b) M2 $f=60.5 \mathrm{~Hz}$. (c) M3 $f=83.0 \mathrm{~Hz}$. (d) $\mathrm{M} 4 f=91.0 \mathrm{~Hz}$. (e) M5 $f=108.1 \mathrm{~Hz}$. (f) M6 $f=145.8 \mathrm{~Hz}$. 


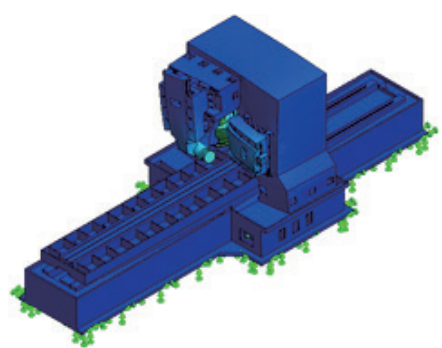

(g)

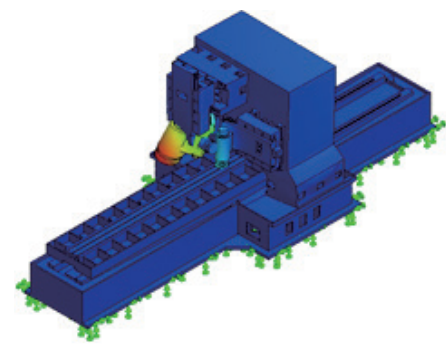

(h)

Fig. 6. (Color online) (Continued) First eight mode shapes in Case 4. (g) M7 $f=146.8 \mathrm{~Hz}$. (h) M8 $f=150.6 \mathrm{~Hz}$.

seen that the first mode shape occurs at $40 \mathrm{~Hz}$, with forward and backward vibration; this frequency is about 1.11 times that in Case 1 . In the frequently used cutting range of 0-3000 rpm $(0-50 \mathrm{~Hz})$, this specific frequency $(40 \mathrm{~Hz})$ still has a high possibility of inducing resonance. The machine user may make some adjustments accordingly, such as avoiding operation near this frequency.

Furthermore, the maximal dynamic stiffness distribution of the machine structure in Case 4 is obtained via FEA and its results are shown in Table 9. In the frequently used cutting range $(0-500 \mathrm{~Hz})$, two large displacements of 0.148 and $0.106 \mathrm{~mm}$ appear at 40.0 and $149.7 \mathrm{~Hz}$, respectively. These two frequencies under external stimuli would cause a fatal error in machining. Compared with the corresponding values of the prototype in Case $1(0.486 \mathrm{~mm}$ at $35.9 \mathrm{~Hz}$ and $0.378 \mathrm{~mm}$ at $148.4 \mathrm{~Hz}$ ) as well as those in the other six cases, the two peak values in Case 4 are much smaller. Among the eight cases, the best dynamic stiffness is found in Case 4.

On the other hand, a verification experiment using displacement sensors is performed to examine the static stiffness of the obtained optimal structure of the CNC grinding machine in Case 4. The same experimental conditions are adopted as for Case 1. The measured maximal static deformation is $0.0143 \mathrm{~mm}$ and occurs at the bottom of the upper grinding head, which corresponds to an error of $8.47 \%$ relative to the simulation result $(0.0132 \mathrm{~mm})$.

Furthermore, a comparison of the results of this study with those of a similar study for the same target machine is made as follows. In the previous study by Wang et al., ${ }^{(28)}$ the target CNC grinding machine based on the optimization of static stiffness exhibited good static behavior with a maximum static deformation of $0.0131 \mathrm{~mm}$, while in this study, it exhibits slightly worse behavior with a maximum static deformation of $0.0143 \mathrm{~mm}$, about $9 \%$ larger. In contrast, the optimal target structure in Ref. 28 showed a dynamic deformation of $0.486 \mathrm{~mm}$ at $35.9 \mathrm{~Hz}$ (similar to that of Case 1 in this study), while in this study, it exhibited better dynamic stiffness with a maximum deformation of $0.148 \mathrm{~mm}$ at $40 \mathrm{~Hz}$, about $30 \%$ smaller. Note that under real cutting conditions, only the dynamic stiffness has a major influence on the final precision of cutting for a machine tool. Therefore, an optimal structure obtained by the consideration of both static and dynamic stiffness is a better choice. 
Table 9

Maximal displacements for different frequencies in dynamic response of structure in Case 4.

\begin{tabular}{|c|c|c|c|c|c|c|c|}
\hline$f(\mathrm{~Hz})$ & $\delta(\mathrm{mm})$ & $f$ & $\delta$ & $f$ & $\delta$ & $f$ & $\delta$ \\
\hline $1.59 \mathrm{E}-06$ & 0.002 & 251.6 & 0.018 & 360.1 & 0.002 & 450.4 & 0.001 \\
\hline 40.0 & 0.148 & 260.2 & 0.004 & 365.6 & 0.001 & 459.2 & 0.001 \\
\hline 55.7 & 0.003 & 265.4 & 0.003 & 373.1 & 0.001 & 467.3 & 0.001 \\
\hline 80.8 & 0.004 & 270.8 & 0.003 & 380.9 & 0.001 & 470.1 & 0.001 \\
\hline 90.1 & 0.005 & 280.1 & 0.006 & 393.6 & 0.001 & 472.3 & 0.001 \\
\hline 104.1 & 0.007 & 287.6 & 0.002 & 401.1 & 0.001 & 473.3 & 0.001 \\
\hline 136.9 & 0.007 & 290.8 & 0.005 & 404.5 & 0.001 & 479.5 & 0.001 \\
\hline 146.5 & 0.023 & 293.7 & 0.003 & 409.6 & 0.002 & 483.0 & 0.002 \\
\hline 149.7 & 0.106 & 297.7 & 0.002 & 416.0 & 0.003 & 487.9 & 0.001 \\
\hline 153.4 & 0.031 & 303.7 & 0.003 & 420.4 & 0.005 & 491.8 & 0.001 \\
\hline 166.7 & 0.004 & 308.3 & 0.003 & 424.3 & 0.003 & 495.2 & 0.001 \\
\hline 198.1 & 0.002 & 311.0 & 0.004 & 431.6 & 0.001 & 498.0 & 0.001 \\
\hline 212.2 & 0.016 & 318.5 & 0.014 & 436.1 & 0.002 & 499.9 & 0.003 \\
\hline 220.1 & 0.006 & 332.4 & 0.004 & 441.8 & 0.001 & 500.4 & 0.003 \\
\hline 229.2 & 0.005 & 337.9 & 0.019 & 445.1 & 0.001 & - & - \\
\hline 235.4 & 0.002 & 345.4 & 0.004 & 447.2 & 0.001 & - & - \\
\hline 246.4 & 0.001 & 351.8 & 0.003 & 449.5 & 0.001 & - & - \\
\hline
\end{tabular}

\section{Concluding Remarks}

In this study, we proposed a novel integral-stiffness-based method to efficiently and accurately explore the optimal structure of a CNC three-head grinding machine. Starting from a prototype structure designed on the basis of know-how, we examined its static and dynamic weaknesses and obtained possible changes of the structure's dimensions for optimization. Then, with the adoption of Taguchi's OA of L8 $\left(2^{7}\right)$, which uses the static stiffness, first natural frequency, and dynamic stiffness as target factors, we performed eight FEA numerical experiments. Also, through GRA, it was found that Case 4 had the optimal integral stiffness (both static and dynamic stiffness) structure. Although Case 5 had the best static stiffness, it does not necessarily have the best final precision during machining. We proved that the dynamic behaviors of a machine's structure are a significant factor that influences the final deformation during machining. Simultaneously considering the static and dynamic stiffness of the machine's structure is a better and more accurate way to optimize the high-stiffness structure of CNC machine tools.

\section{Acknowledgments}

This work was carried out as part of the Intelligent Manufacturing Program coordinated by the Beautiful China Research Institute of Sanming University with support by Operational Funding of the Advanced Talents for Scientific Research (19YG05) supported by Sanming University. We also acknowledge support from the College of Mechanical and Electrical Engineering, Sanming University. 


\section{References}

1 C. Liu, T. Feng, L. Wang, and C. Zhaoyong: J. Mech. Eng. 52 (2016) 161. https://doi.org/10.3901/ JME.2016.03.161

2 Z. Ting: Forg. Stamp. Technol. 35 (2010) 74.

3 Y. Altintas and Y. Cao: CIRP Annals. 54 (2005) 379. https://doi.org/10.1016/S0007-8506(07)60127-9

4 C. P. Reddy and S. S. Rao: J. Eng. Indus. 100 (1978) 137. https://doi.org/10.1115/1.3439401

5 S. S. Rao and R. V. Grandhi: J. Mech. Trans. Autom. 105 (1983) 236. https://doi.org/10.1115/1.3258515

6 S. J. Lee and S. G. Kapoor: J. Eng. Optim. 10 (1986) 25. https://doi.org/10.1080/03052158608902525

7 T. Prakosa, A. Wibowo, and R. Ilhamsyah: J. Kones 20 (2013) 363. https://doi.org/10.5604/12314005.1137848

8 G. Lei, Z. Hui, and Y. Peiqing: J. Tsingh. Univ. 51 (2011) 846.

9 D. Chen, X. Lu, and X. Sun: Proc. CIRP 78 (2018) 279. https://doi.org/10.1016/j.procir.2018.08.305

10 R. Liusheng, H. Liang, and P. Yongjun: Mach. Des. Res. 26 (2010) 87.

11 K. Jie: J. Hum. Univ. Arts Sci. 26 (2014) 46.

12 W. H. Yang and Y. S. Tarng: J. Mater. Proc. Technol. 84 (1998) 122. https://doi.org/10.1016/S09240136(98)00079-X

13 S. Moaveni: Finite Element Analysis, Theory, and Application with ANSYS (Prentice Hall, New York, 2014) 4th ed., Chap. 5.

14 R. Mahdavinejad: Int. J. Mach. Tools Manuf. 45 (2005) 753. https://doi.org/10.1016/j.ijmachtools.2004.11.017.

15 J. J. Wu: Int. J. Mech. Sci. 46 (2004) 1245. https://doi.org/10.1016/j.ijmecsci.2004.07.002

16 D. T. Y. Huang and J. J. Lee: Int. J. Mach. Tools Manuf. 41 (2001) 1149. https://doi.org/10.1016/s08906955(01)00012-8J

17 S. Yang: Int. J. Mach. Tool Des. Res. 21 (1981) 23. https://doi.org/10.1016/0020-7357(81)90011-1

18 J. Majerik and J. Jambo: Procedia Eng. 100 (2015) 450. https://doi.org/10.1016/j.proeng.2015.01.390

19 E. Wagner: Procedia Technol. 19 (2015) 34. https://doi.org/10.1016/j.protcy.2015.02.006

20 D. Kano, T. Lorenzer, S. Weikert, and K. Wegener: Prec. Eng. 34 (2010) 399. https://doi.org/10.1016/j. precisioneng.2009.09.003

21 J. J. Wu: Measurement 39 (2006) 740. https://doi.org/10.1016/j.measurement.2006.03.002

22 Y. Kang, Y. P. Chang, J. W. Tsai, S. C. Cheng, and L. K. Yang: Fin. Elem. Anal. Des. 37 (2001) 485. https://doi. org/2001.0.1016/S0168-874X(00)00049-4.

23 K. Vivekananda, G. N. Arka, and S. K. Sahoo: Pocedia Mater. Sci. 6 (2014) 1906. https://doi.org/10.1016/j. mspro.2014.07.22314.

24 C. C. Hong, C. L. Chang, and C. Y. Lin: Eng. Sci. Technol. 19 (2016) 1971. https://doi.org/10.1016/j. jestch.2016.07.013

25 K. C. Wang: Eng. Comput. 31 (2014) 1761. https://doi.org/10.1108/ec-11-2012-0296.

26 W. H. Yang and Y. S. Tarng: J. Mater. Proc. Tech. 84 (1998) 122. https://doi.org/10.1016/S09240136(98)00079-X14

27 J. L. Deng: J. Grey Syst. 1 (1984) 1.

28 K. C. Wang, C. H. Yang, L. Wu, and H. Gao: Sens. Mater. 32 (2020) 1633. https://doi.org/10.18494/ SAM.2020.2681 